\title{
Sildenafil Reduces the Risk of Thromboembolic Events in HeartMate II Patients with Low-Level Hemolysis and Significantly Improves the Pulmonary Circulation
}

\author{
Rashad Zayat, ${ }^{1}$ MD, Usaama Ahmad, ${ }^{1}$ MD, Christian Stoppe, ${ }^{2}$ PhD, Mohammad Amen Khattab, ${ }^{1}$ MD, \\ Fateh Arab, ${ }^{3} \mathrm{MD}$, Ajay Moza, ${ }^{1} \mathrm{PhD}$, Lachmandath Tewarie, ${ }^{1} \mathrm{MD}$, Andreas Goetzenich, ${ }^{1} \mathrm{PhD}$, \\ Rüdiger Autschbach, ${ }^{1} \mathrm{PhD}$ and Heike Schnoering, ${ }^{1} \mathrm{PhD}$
}

\begin{abstract}
Summary
Low-level hemolysis (LLH) after left ventricular assist device implantation contributes to thromboembolic events (TE). Free plasma hemoglobin ( $\mathrm{fHb}$ ) scavenges nitric oxide (NO), which causes endothelial dysfunction and activates platelets. fHb also interacts with von Willebrand factor (vWF). We hypothesized that improved hemodynamic and enhanced NO signaling in HeartMate II (HMII) patients with LLH taking the phosphodiesterase-5 inhibitor sildenafil may reduce the risk of TE.

From 2011 to 2015, 83 patients underwent HMII implantation. Patients with LLH as defined by elevated lactate dehydrogenase $(400<\mathrm{LDH} \leq 700 \mathrm{U} / \mathrm{L})$ at hospital discharge were identified. Patients were categorized into 4 groups: 1) LLH + sildenafil, 2) LLH no sildenafil, 3) no LLH + sildenafil, and 4) no LLH no sildenafil. Adverse event-free survival was compared between the groups.

Thirty-four patients (40.9\%) were discharged with LLH and $22(64.7 \%)$ of them took sildenafil. LDH and $\mathrm{fHb}$ remained significantly elevated in both LLH groups compared to the no LLH patients $(P<0.0001)$. Overall incidence of pump thrombosis (PT) was $4.8 \%$ and of ischemic stroke (IS) was $8.4 \%$. HMII patients with LLH not on sildenafil had higher risk of TE (hazard ratio (HR): $14.4,95 \%$-CI: $1.8-117.1, P=0.001$ ). vWF activity and bleeding incidence did not differ between the LLH and no LLH patients. Mean pulmonary artery pressure and pulmonary vascular resistance decreased significantly in HMII taking sildenafil $(P<0.0001)$ while cardiac index increased $(P<0.0001)$.

Sildenafil treatment among HMII patients with LLH reduced the risk of thromboembolic events and significantly improved and decompressed the pulmonary circulation during HMII support.

(Int Heart J 2018; 59: 1227-1236)

Key words: Free plasma hemoglobin, Lactate dehydrogenase, Phosphodiesterase 5 inhibitors, Left ventricular assist devices, Pump thrombosis, Stroke, Nitric oxide
\end{abstract}

$\mathrm{C}$ ontinuous-flow (CF) left ventricular assist device (LVAD) support is a common form of treatment for end-stage heart failure with clear evidence of improving survival and quality of life. ${ }^{1,2)}$ The number of implanted CF-LVADs as destination therapy is increasing. ${ }^{1)}$ Consequently, the prevalence of device-related complications is also increasing. ${ }^{3)}$ Hemolysis represents one of the most common complications after CF-LVAD implantation with an estimated incidence of $15 \%$ to $30 \%,{ }^{4,5)}$ and it has been linked to pump thrombosis (PT), peripheral embolism, and higher morbidity and mortality. ${ }^{67)}$ The Interagency Registry of Mechanically Assisted Circulatory Support (INTERMACS) defines hemolysis as elevated free plasma hemoglobin ( $\mathrm{fHb}>40 \mathrm{mg} / \mathrm{dL}$ ) accompanied with clinical signs. ${ }^{8)}$ Another accepted definition of hemo- lysis after LVAD implantation is a rise in lactate dehydrogenase $(\mathrm{LDH})$ levels to $>2.5$ the upper limit of normal values (> $700 \mathrm{U} / \mathrm{L}){ }^{9}{ }^{9}$ A study by Shah, et al. also demonstrated that LDH is a superior marker in detecting hemolysis in LVAD patients. ${ }^{10)}$ The epidemiology of hemolysis in LVAD patients and the consequences of a hemolytic event are still incompletely understood. The released $\mathrm{fHb}$ due to erythrocyte destruction during hemolysis can directly activate platelets and induce a prothrombotic state. ${ }^{11)}$ Similar phenomena have been detected in patients with severe aortic stenosis. ${ }^{12)}$ Previous studies have demonstrated that $\mathrm{fHb}$ scavenges nitric oxide (NO) and reduces the bioavailability of NO during hemolysis, thus leading to endothelial dysfunction and platelet activation. ${ }^{13,14)} \mathrm{At}$ the same time, elevated $\mathrm{fHb}$ interacts with the $\mathrm{A} 1$ and 2

From the ${ }^{1}$ Department of Thoracic and Cardiovascular Surgery, RWTH University Hospital, Aachen, Germany, ${ }^{2}$ Department of Intensive Care Medicine, RWTH University Hospital, Aachen, Germany and ${ }^{3}$ Department of Cardiovascular Medicine, Dr. Hamid Center, Dubai Health City, United Arab Emirates.

Address for correspondence: Rashad Zayat, MD, Department of Thoracic and Cardiovascular Surgery, RWTH University Hospital, Pauwelsstrasse 30, 52074 Aachen, Germany. E-mail: rzayat@ukaachen.de

Received for publication January 1, 2018. Revised and accepted March 2, 2018

Released in advance online on J-STAGE October 10, 2018.

doi: 10.1536/ihj.18-001

All rights reserved by the International Heart Journal Association. 
domains of von Willebrand factor (vWF), which increases platelet adhesion to $\mathrm{vWF}^{15)}$

Phosphodiesterase-5-inhibitors (PDE5-Is), such as sildenafil, enhance NO signaling and, therefore, the effect of NO. ${ }^{16,17)}$ PDE5-I have also been used during LVAD support to prevent right ventricular dysfunction or to improve it through decreases of pulmonary artery pressure and pulmonary vascular resistance (PVR) and therefore providing a higher cardiac output, and previous studies demonstrated the beneficial effect of PDE5-I use during LVAD support. $^{18-20)}$

We hypothesized that sildenafil might reduce the risk of thromboembolic events (TE) in HeartMate II (HMII) patients with low-level hemolysis (LLH), as defined by elevated $\mathrm{LDH}(400<\mathrm{LDH} \leq 700 \mathrm{U} / \mathrm{L})$.

\section{Methods}

After obtaining informed consent from the patient and approval by the local ethics committee (Ethikkommission der RWTH Aachen, ethical vote no 151/09), blood samples were taken at predefined time points to perform additional analyses. Clinical data were retrospectively collected.

Study design and data collection: We conducted a single-center, retrospective, case-control study of patients who underwent HMII implantation during a 4-year period from April 2011 to April 2015. Patients were excluded from this analysis if they did not survive to discharge during the index hospitalization for LVAD implantation. Baseline demographic, baseline hemodynamic parameters from right heart catheterization and from echocardiography, and peri- and postoperative data were collected from our institution's database. Laboratory data (e.g., LDH, international normalized ratio (INR), fHb, hemoglobin ( $\mathrm{Hb}$ ), aspartate transaminase (AST), alanine transaminase (ALT), total bilirubin and N-terminal pro b-type natriuretic peptide (NT-proBNP) were retrieved. $\mathrm{fHb}$ was not measured preoperatively. $\mathrm{vWF}$ antigen (vWF:Ag) as well as its collagen binding activity (vWF:CBA) and VWF ristocetin cofactor activity (vWF:RCo) were measured 3 months postoperatively. vWF multimer analysis was also performed 3 months postoperatively in a reference external laboratory. All patients were routinely followed-up in our outpatient clinic until July 2017. During follow-up visits patients had transthoracic echocardiography (TTE) studies to evaluate the right and left ventricular function (RV and LV). Additionally, almost all LVAD patients in our institution receive hemodynamic reevaluation 1 year after LVADimplantation with right heart catheterization or a SwanGanz catheter to optimize the treatment and update the status if needed.

During the study period, our institutional anticoagulation protocol for HMII patients was to administer aspirin (100 mg) and phenprocoumon, a Vitamin-Kantagonist, with a goal INR of 1.8-2.2 after implant.

Adverse event monitoring: Each patient's history of adverse events (AE) history was evaluated during the inpatient treatment and at every outpatient visit. AEs were defined according to the 2013 INTERMACS Version 2.2 definitions for ischemic stroke (IS), hemorrhagic stroke
(HS), PT, bleeding and hemolysis. ${ }^{8)}$ IS or HS were defined by the presence of an acute cerebral infarction/ bleeding noted on a cranial computed tomographic scan and accompanied by neurological symptoms. Signs and symptoms of suspected PT included at least 2 of the 3 following criteria: 1) Presence of hemolysis, 2) Presence of heart failure not explained by structural heart disease, 3) Abnormal pump parameters. PT was confirmed via direct visual inspection or by incontrovertible contrast radiographic evidence or by the absence of an appropriate Doppler flow signal that results in or could potentially induce circulatory failure caused by thromboembolism. Gastrointestinal bleeding (GIB) was defined by a drop in hemoglobin of $>1 \mathrm{~g} / \mathrm{dL}$, requiring a blood transfusion and endoscopic detection of bleeding source or angiodysplastic lesions of the gastrointestinal tract.

Patients groups: Patients with elevated LDH $(400<\mathrm{LDH}$ $\leq 700 \mathrm{UL}$ ) (normal range in our laboratory is $<280 \mathrm{U} / \mathrm{L}$ ) at hospital discharge were identified. Patients were then categorized into 1 of 4 groups; group 1 taking sildenafil and had LLH (LLH + sildenafil), group 2 had LLH and were not taking sildenafil (LLH no sildenafil), group 3 did not have LLH and were on sildenafil (no LLH + sildenafil), and group 4 did not have LLH and were not on sildenafil (no LLH no sildenafil). If one of the following criteria was met, sildenafil was then prescribed after LVAD implantation on postoperative day 1: 1) mean pulmonary artery pressure (mPAP) $>25 \mathrm{mmHg}$; 2) pulmonary capillary wedge pressure $(\mathrm{PCWP})>15 \mathrm{mmHg}$; 3) PVR > 1.6 Wood units; 4) tricuspid annular plane systolic excursion (TAPSE) $<14 \mathrm{~mm}$; or 5) right ventricular fractional area change $(\mathrm{RV}-\mathrm{FAC})<30 \%$.

Statistical analysis: Continuous data were assessed for normality using the Kolmogorov-Smirnov test, and then presented as the mean \pm standard deviation or median (minimum, maximum). Comparison of repeated continuous measurements between groups was performed by twoway ANOVA test or the Kruskal-Wallis test, as appropriate. For the comparison of continuous parameters without repeated measurements one-way ANOVA test, or multiple $t$-test were used, as appropriate.

Categorical variables are presented as frequencies, and differences were tested by Fisher's exact or the Chisquare test, as appropriate. Survival analysis was performed using Kaplan-Meier curves with the log-rank signed test, with time 0 being the date of implantation. Patients were censored for death, heart transplantation or device explant due to recovery. Cox regression modeling was used to calculate hazard ratios (HRs) and 95\% confidence intervals [95\% CIs] for events in groups. All data analyses were performed using SPSS 24 (IBM Corp., Armonk, NY, USA). A $P$-value $<0.05$ was considered statistically significant.

\section{Results}

This study included 83 patients who underwent HMII implantation at our institution and survived to discharge from the index hospitalization. Mean age was $62.6 \pm 8.5$ years and 13 patients $(15.7 \%)$ were female. The median (minimum, maximum) patient support with HMII among 
Table I. Patient Characteristics and Preoperative Laboratory and Perioperative Data

\begin{tabular}{|c|c|c|c|c|c|}
\hline & $\begin{array}{c}\text { LLH + Sildenafil } \\
\quad(n=22)\end{array}$ & $\begin{array}{l}\text { LLH no Sildenafil } \\
\quad(n=12)\end{array}$ & $\begin{array}{c}\text { no LLH + Sildenafil } \\
\quad(n=34)\end{array}$ & $\begin{array}{l}\text { no LLH no Sildenafil } \\
\qquad(n=15)\end{array}$ & $P$ value \\
\hline Age, years & $60.8 \pm 10.7$ & $61.2 \pm 7.7$ & $63.7 \pm 8.2$ & $63.8 \pm 5.6$ & 0.554 \\
\hline Female $n(\%)$ & $5(22.7)$ & $3(25)$ & $3(8.8)$ & $2(13.3)$ & 0.368 \\
\hline BMI kg/m² & $28.1 \pm 5.8$ & $27.5 \pm 3.5$ & $26.5 \pm 3.5$ & $26.1 \pm 8.3$ & 0.427 \\
\hline $\mathrm{HT} n(\%)$ & $15(68.2)$ & $8(66.7)$ & $27(79.4)$ & $12(80)$ & 0.770 \\
\hline $\operatorname{IDDM} n(\%)$ & $2(9.1)$ & $1(8.3)$ & 7 (20.6) & 0 & 0.202 \\
\hline $\operatorname{PAD} n(\%)$ & $4(18.1)$ & $3(25)$ & $10(29.4)$ & $4(26.7)$ & 0.811 \\
\hline $\operatorname{ICM} n(\%)$ & $17(77.3)$ & $9(75)$ & $25(73.5)$ & $13(86.7)$ & 0.764 \\
\hline $\operatorname{DCM} n(\%)$ & $5(22.7)$ & $3(25)$ & $9(26.5)$ & $2(13.3)$ & 0.764 \\
\hline PHT $n(\%)$ & 18 & 0 & 26 & 0 & 0.0001 \\
\hline $\mathrm{EF} \%$ & $20.8 \pm 7.6$ & $20.5 \pm 7.8$ & $18 \pm 4.3$ & $20.7 \pm 5.5$ & 0.275 \\
\hline Prior dialysis $n(\%)$ & 0 & $1(8.3)$ & $1(2.9)$ & 0 & 0.391 \\
\hline Prior cardiac surgery $n(\%)$ & $6(27.3)$ & $1(8.3)$ & $3(8.8)$ & $5(33.3)$ & 0.106 \\
\hline \multicolumn{6}{|l|}{ INTERMACS level } \\
\hline I & $5(22.8)$ & $4(33.3)$ & 0 & $3(20)$ & 0.002 \\
\hline II & $1(4.5)$ & $2(16.7)$ & $5(14.7)$ & $3(20)$ & 0.481 \\
\hline III & 0 & $1(8.3)$ & $4(11.8)$ & $2(13.3)$ & 0.297 \\
\hline IV & $16(72.7)$ & $5(41.7)$ & $25(73.5)$ & $7(46.7)$ & 0.314 \\
\hline BTT & $4(18.8)$ & $3(25)$ & $8(23.5)$ & $4(26.7)$ & 0.708 \\
\hline DTT & $18(81.2)$ & $9(75)$ & $26(76.5)$ & $11(73.3)$ & 0.708 \\
\hline \multicolumn{6}{|l|}{ Preop laboratory } \\
\hline Creatinine mg/dL & $1.1 \pm 0.3$ & $1.5 \pm 2.2$ & $1.2 \pm 0.5$ & $1.1 \pm 0.4$ & 0.484 \\
\hline NT-proBNP ng/L & $3192(614,9059)$ & $5236(4780,5692)$ & $4884(965,9792)$ & $1092(820,1365)$ & 0.121 \\
\hline LDH U/L & $226(175,939)$ & $233(148,686)$ & $213(148,686)$ & $196(124,491)$ & 0.349 \\
\hline $\mathrm{Hb} \mathrm{g} / \mathrm{dL}$ & $13.1(8.7,16.7)$ & $10.9(8,16)$ & $13.1(9,15.9)$ & $12.7(7.8,16)$ & 0.880 \\
\hline Platelet count $\times 10^{3}$ & $199(36,345)$ & $194.5(63,317)$ & $221(92,468)$ & $211.5(92,475)$ & 0.351 \\
\hline AST U/L & $40(18,213)$ & $32(20,130)$ & $26(16,92)$ & $29.5(13,80)$ & 0.011 \\
\hline ALT U/L & $48(16,159)$ & $39.5(16,91)$ & $28.5(11,111)$ & $24.5(14,71)$ & 0.011 \\
\hline Total bilirubin & $1.5 \pm 1.4$ & $0.9 \pm 0.6$ & $1.1 \pm 1.1$ & $1.1 \pm 0.9$ & 0.419 \\
\hline INR & $1.2 \pm 0.40$ & $1.1 \pm 0.08$ & $1.2 \pm 0.3$ & $1.1 \pm 0.2$ & 0.268 \\
\hline MPAP mmHg & $30.2 \pm 8.9$ & $22.6 \pm 3.8$ & $34.3 \pm 9.7$ & $21.4 \pm 7.7$ & $<0.0001$ \\
\hline PCWP mmHg & $22.2 \pm 6.3$ & $16.1 \pm 1.5$ & $21.1 \pm 9.3$ & $14.3 \pm 5.6$ & 0.0001 \\
\hline PVR Wood units & $2.5 \pm 0.8$ & $1.4 \pm 0.4$ & $3.0 \pm 1.1$ & $1.5 \pm 0.9$ & $<0.0001$ \\
\hline $\mathrm{CI} \mathrm{L} / \mathrm{minute} / \mathrm{m}^{2}$ & $2.0 \pm 0.4$ & $2.1 \pm 0.3$ & $1.9 \pm 0.4$ & $1.9 \pm 0.3$ & 0.905 \\
\hline TAPSE $\mathrm{cm} / \mathrm{second}$ & $15.3 \pm 4.4$ & $16.8 \pm 2.1$ & $14.3 \pm 2.3$ & $16.2 \pm 2.8$ & 0.066 \\
\hline RV-FAC \% & $36.9 \pm 10.2$ & $39.4 \pm 9.4$ & $35.4 \pm 9.2$ & $40.3 \pm 9.6$ & 0.348 \\
\hline \multicolumn{6}{|l|}{ Peri-op data } \\
\hline HMII alone & $10(45.5)$ & $6(50.0)$ & $13(38.0)$ & $7(46.7)$ & 0.797 \\
\hline $\mathrm{HMII}+\mathrm{CABG}$ & $9(40.9)$ & $4(33.3)$ & $11(32.4)$ & $5(33.3)$ & 0.928 \\
\hline HMII + TVR & $1(4.5)$ & $1(8.3)$ & $5(14.7)$ & $2(13.3)$ & 0.117 \\
\hline $\mathrm{HMII}+\mathrm{CABG}+$ Valve & $2(9.1)$ & $1(8.3)$ & $5(14.7)$ & $1(6.7)$ & 0.884 \\
\hline
\end{tabular}

AST indicates aspartate transaminase; ALT, alanine transaminase; BMI, body mass index; CABG, coronary artery bypass grafting; CBP, cardiopulmonary bypass; CI, cardiac index; Hb, hemoglobin; HMII, HeartMate II; HT, arterial hypertension; ICM, ischemic cardiomyopathy; IDDM, insulin-dependent diabetes mellitus; INTERMACS, Interagency Registry for Mechanically Assisted Circulatory Support; LDH, lactate dehydrogenase; NT-proBNP, N-terminal prohormone of brain natriuretic peptide; PAD, peripheral arterial disease; PHT, pulmonary hypertension; preop, preoperative; PCWP, pulmonary capillary wedge pressure; TVR, tricuspid valve replacement; TAPSE, tricuspid annular plane systolic excursion; EF, ejection fraction; DCM, dilated cardiomyopathy; MPAP, mean pulmonary artery pressure; PVR, pulmonary vascular resistance; and RV-FAC, right ventricular fractional area change.

all 83 HMII patients was 28 months (2, 83 months). The incidence of $\mathrm{AE}$ during follow-up in the study cohort $(83$ patients) was as follows: $16.9 \%$ hemolysis by INTERMACS definition with 0.10 events per patient-year (eppy); $4.8 \%$ PT (0.018 eppy); $8.4 \%$ IS (0.032 eppy); 6\% TIA (0.023 eppy); $4.9 \%$ HS (0.018 eppy); and $45.1 \%$ GIB (0.59 eppy).

A total of $34(40.9 \%)$ patients were discharged with LLH with a median LDH of $478(410,700)$ U/L. Among those patients, $22(64.7 \%)$ had a median LDH of 483 $(410,700) \mathrm{U} / \mathrm{L}$ and were discharged with sildenafil treatment $(\mathrm{LLH}+$ sildenafil group) and 12 patients $(35.3 \%)$ had a median LDH of $457(411,700)$ U/L and were not taking sildenafil (LLH no sildenafil group). Baseline characteristics, preoperative laboratory, demographics, and operative data of all 4 groups are presented in Table I. Significantly more patients had INTERMACS level I in the LLH no sildenafil group than patients in the other 3 groups $(P=0.002)$. Regarding preoperative laboratory, only AST and ALT differed significantly between groups. AST was significantly higher in the LLH + sildenafil group compared to the no LLH + sildenafil and no LLH no sildenafil groups, $40(18,213)$ versus $26(16,92) \mathrm{U} / \mathrm{L}$, $P<0.001$ and $40(18,213)$ versus $29.5(13,80) \mathrm{U} / \mathrm{L}, P=$ 
Table II. Time Course of Hemolysis and Liver Function Markers in the 4 Study Groups

\begin{tabular}{|c|c|c|c|c|c|c|c|c|}
\hline & $\begin{array}{l}\text { 1. LLH + } \\
\text { Sildenafil } \\
(n=22)\end{array}$ & $\begin{array}{l}\text { 2. LLH no } \\
\text { Sildenafil } \\
(n=12)\end{array}$ & $\begin{array}{c}P \text {-value } \\
1 \text { versus } 2\end{array}$ & $\begin{array}{c}P \text {-value } \\
1 \text { versus } 3\end{array}$ & $\begin{array}{l}\text { 3. no LLH + } \\
\text { Sildenafil } \\
(n=34)\end{array}$ & $\begin{array}{c}P \text {-value } \\
2 \text { versus } 3\end{array}$ & $\begin{array}{l}\text { 4. no LLH no } \\
\text { Sildenafil } \\
(n=15)\end{array}$ & $\begin{array}{c}P \text {-value } \\
3 \text { versus } 4\end{array}$ \\
\hline \multicolumn{9}{|c|}{ Lactate dehydrogenase U/L } \\
\hline Pre-op U/L & $226(175,939)$ & $233(148,686)$ & 0.703 & $0.004 *$ & $213(148,686)$ & 0.314 & $196(124,491)$ & 0.998 \\
\hline at discharge $\mathrm{U} / \mathrm{L}$ & $483(410,700)$ & $457(411,700)$ & 0.999 & $<0.0001 *$ & $319(160,377)$ & $<0.001 *$ & $338(15,380)$ & 0.996 \\
\hline 3 months U/L & $409(301,575)$ & $571(433,840)$ & $0.001 *$ & 0.511 & $312(211,1492)$ & $<0.001 *$ & $302(217,580)$ & 0.763 \\
\hline 6 months U/L & $423(313,540)$ & $474.5(338,908)$ & $0.036^{*}$ & 0.301 & $324(78,893)$ & $<0.001 *$ & $285(173,602)$ & 0.648 \\
\hline 12 months U/L & $374(272,580)$ & $521(330,840)$ & $0.006^{*}$ & 0.287 & $350(225,460)$ & $<0.001 *$ & $350(225,460)$ & 0.837 \\
\hline \multicolumn{9}{|c|}{ Free plasma hemoglobin $\mathrm{mg} / \mathrm{dL}$} \\
\hline at discharge $\mathrm{mg} / \mathrm{dL}$ & $45(7,71)$ & $45(33,67)$ & 0.999 & $<0.0001 *$ & $20(9.3,77)$ & $<0.0001^{*}$ & $20(10,59)$ & 0.975 \\
\hline 3 months $\mathrm{mg} / \mathrm{dL}$ & $41.5(17,71)$ & $48(44,107)$ & 0.076 & $<0.0001^{*}$ & $18(5.3,86)$ & $<0.0001 *$ & $27.9(15.6,69.2)$ & 0.512 \\
\hline 6 months mg/dL & $26(11,66)$ & $40.5(25.4,84)$ & $0.017 *$ & 0.849 & $26.7(11,83)$ & $0.0009 *$ & $25.2(11,76.2$ & 0.923 \\
\hline 12 months mg/dL & $31.5(11,55)$ & $44.4(12,71)$ & $0.041 *$ & 0.953 & $24(12,93)$ & $0.006^{*}$ & $16(6.2,59.7)$ & 0.839 \\
\hline \multicolumn{9}{|l|}{ Total bilirubin $\mathrm{mg} / \mathrm{dL}$} \\
\hline Pre-op mg/dL & $1.3(0.2,5.9)$ & $0.8(0.2,2.5)$ & 0.325 & 0.245 & $0.6(0.3,5.6)$ & 0.961 & $0.8(0.2,3.6)$ & 0.991 \\
\hline at discharge $\mathrm{mg} / \mathrm{dL}$ & $0.6(0.2,2.2)$ & $0.6(0.3,2.2)$ & 0.974 & 0.966 & $0.6(0.2,2.7)$ & 0.865 & $0.5(0.2,2.2)$ & 0.907 \\
\hline 3 months $\mathrm{mg} / \mathrm{dL}$ & $0.6(0.2,3.9)$ & $0.9(0.2,3.9)$ & 0.833 & 0.969 & $0.5(0.2,3.8)$ & 0.577 & $0.6(0.3,5.5)$ & 0.972 \\
\hline 6 months mg/dL & $0.4(0.2,2.8)$ & $1.3(0.3,2.1)$ & 0.957 & 0.686 & $0.5(0.2,2.1)$ & 0.469 & $1.2(0.3,2.1)$ & 0.643 \\
\hline 12 months mg/dL & $0.5(0.2,3.9)$ & $1.2(0.4,2.1)$ & 0.485 & 0.662 & $1.1(0.2,3.3)$ & 0.938 & $0.4(0.2,2.4)$ & 0.757 \\
\hline \multicolumn{9}{|c|}{ Alanine aminotransferase U/L } \\
\hline Pre-op U/L & $47(16,159)$ & $39.5(16,91)$ & 0.073 & $0.006 *$ & $28(11,111)$ & 0.903 & $24.5(14,71)$ & 0.893 \\
\hline at discharge U/L & $37(14,136)$ & $30(14,84)$ & 0.636 & $0.028 *$ & $22(8,75)$ & 0.719 & $26.5(14,104)$ & 0.755 \\
\hline 3 months U/L & $17(9,54)$ & $17.5(11,37)$ & 0.979 & 0.956 & $17(8,48)$ & 0.999 & $21.5(13,53)$ & 0.856 \\
\hline 6 months U/L & $25(12,83)$ & $20.5(14,29)$ & 0.652 & 0.594 & $21(8,48)$ & 0.997 & $23(8,60)$ & 0.911 \\
\hline 12 months U/L & $27(15,49)$ & $20.5(11,45)$ & 0.870 & 0.441 & $20(8,42)$ & 0.973 & $19.5(11,43)$ & 0.999 \\
\hline \multicolumn{9}{|c|}{ Aspartate aminotransferase U/L } \\
\hline Pre-op U/L & $40(18,213)$ & $32(20,130)$ & 0.491 & $<0.001 *$ & $26(16,92)$ & $0.045^{*}$ & $29.5(13,80)$ & 0.659 \\
\hline at discharge U/L & $26(22,54)$ & $25(14,134)$ & 0.663 & 0.353 & $25(14,134)$ & 0.998 & $36(15,59)$ & 0.607 \\
\hline 3 months U/L & $27(19,47)$ & $26(16,88)$ & 0.769 & 0.450 & $26(16,88)$ & 0.997 & $27(21,38)$ & 0.996 \\
\hline 6 months U/L & $29(24,55)$ & $28(18,78)$ & 0.963 & 0.405 & $28(18,78)$ & 0.996 & $29(12,52)$ & 0.969 \\
\hline 12 months U/L & $30.5(19,46)$ & $29(12,81)$ & 0.336 & 0.963 & $29(12,81)$ & 0.999 & $27(16,40)$ & 0.967 \\
\hline
\end{tabular}

Data are presented as median (minimum, maximum), comparisons of repeated measurements between groups were conducted with 2-way ANOVA with Tukey's correction. * indicates significance.

0.045, respectively. ALT was also significantly higher in the LLH + sildenafil group compared to both no LLH groups (Table II). Total bilirubin did not differ between the groups at any measured time point (Table II).

Laboratory data: Detailed results of laboratory data at all measured time points are presented in Table II and an overview of $\mathrm{LDH}$ and $\mathrm{fHb}$ time courses are presented in Figure 1. LDH remained significantly elevated over time in the LLH no sildenafil group compared to the no LLH groups $(P<0.0001)$ (Figure 1$)$. Within the LLH groups LDH was significantly lower over time in the LLH + sildenafil group compared to the LLH no sildenafil group ( $P$ $=0.006)$. $\mathrm{LDH}$ values in the $\mathrm{LLH}+$ sildenafil group were significantly higher at discharge compared to both no LLH groups (no LLH + sildenafil and no LLH no sildenafil) and then at 3, 6 and 12 months post HMII implantation LDH in the LLH + sildenafil did not differ significantly compared to no LLH patients either on sildenafil or not on sildenafil (Figure 1 and Table II).

$\mathrm{fHb}$ was also significantly higher over time in both LLH groups compared to the no LLH groups $(P<$ $0.0001)$ and within the LLH groups, fHb was significantly lower in the LLH +sildenafil group compared to the LLH no sildenafil group $(P=0.002)$ (Figure 1 and Table II). INR did not differ between all groups at all measured time points (Figure 2).
vWF and LLH: All patients had an acquired von Willebrand disease (AvWD), as determined by the loss of highmolecular-weight multimers (HMWM) via Western blot and by reduced vWF:CBA/vWF:Ag and vWF:RCo/vWF: $\mathrm{Ag}$ ratios. The values of $\mathrm{vWF}: \mathrm{Ag}, \mathrm{vWF}: \mathrm{CBA}$ and $\mathrm{VWF}$ : RCo in all groups 3 months postoperatively are presented in Table III. All vWF values did not differ significantly between groups.

Outcome by sildenafil status: The mean dose of sildenafil was $60 \pm 15 \mathrm{mg} /$ day. The distribution of $\mathrm{AE}$ in each group is presented in Table IV. Among the patients in the LLH no sildenafil group $25 \%$ had PT and $2.9 \%$ of the patients in the no LLH + sildenafil group had PT, whereas no patients in the LLH + sildenafil and no LLH no sildenafil groups had PT $(P=0.005)$. Among the LLH no sildenafil patients $33.3 \%$ had IS and $8.8 \%$ of the no LLH + sildenafil patients had IS and no IS occurred in the LLH + sildenafil and no LLH no sildenafil groups $(P=0.004)$. The incidence of TIA was $9.1 \%$ in the LLH + sildenafil group, $16.7 \%$ in the LLH no sildenafil group compared to $6.7 \%$ in the no LLH no sildenafil group, and no TIAs in the no LLH + sildenafil group were noted $(P=0.764)$. Comparing all TEs including IS, PT and TIA between HMII patients with LLH $(n=34)$ and HMII patients without LLH $(n=49)$ regardless of sildenafil status, HMII patients with LLH had a significantly higher incidence of 
A

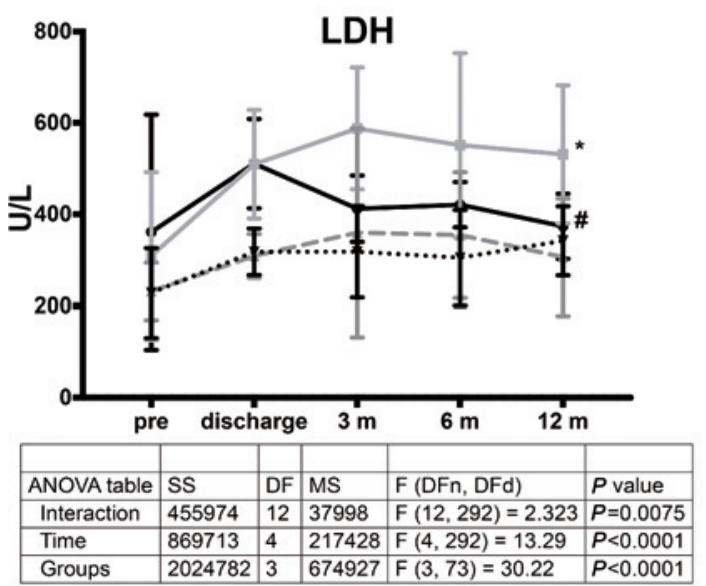

\section{LLH + Sildenafil}

\section{LLH no Sildenafil}

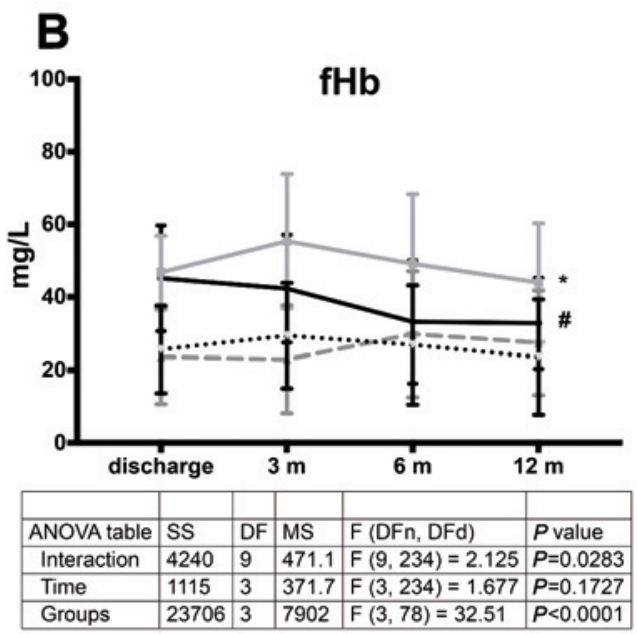

$\rightarrow \cdot$ no LLH + Sildenafil

no LLH no Sildenafil

Figure 1. Time course of lactate dehydrogenase and free plasma hemoglobin. * indicates significance when comparing LLH no sildenafil with no LLH groups $(P<0.0001)$; \# indicates significance when comparing LLH + sildenafil with LLH no sildenafil $(P<$ 0.006). fHb indicates free plasma hemoglobin; LDH, lactate dehydrogenase; preop, preoperative; DF, degrees of freedom; MS, mean square, and F ratio, each F ratio is computed by dividing the MS value by another MS value. The MS value for the denominator depends on the experimental design.

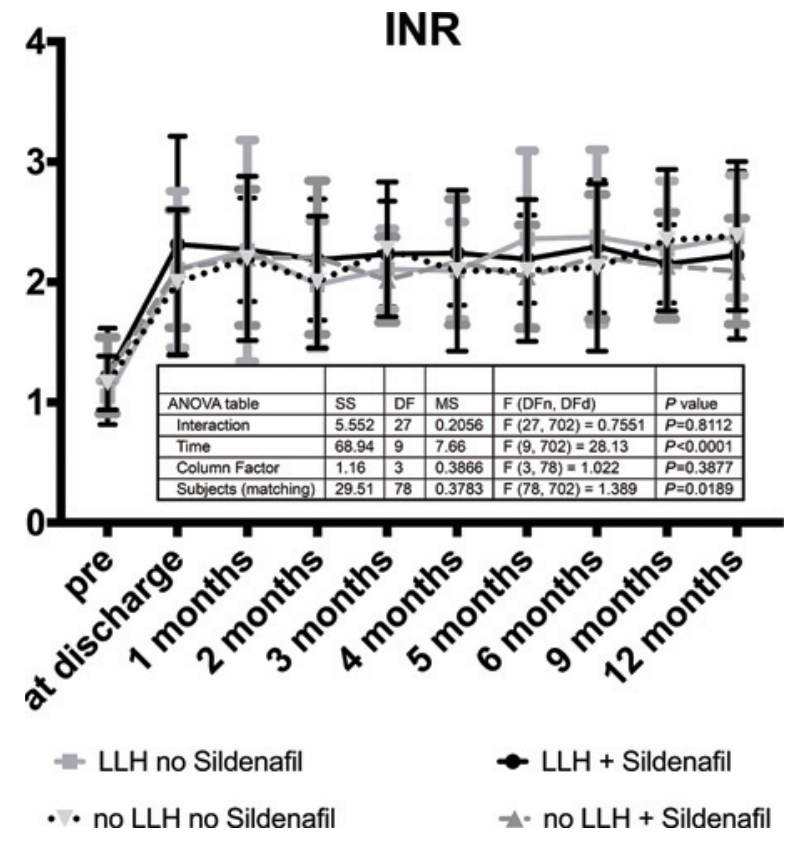

Figure 2. Comparison of INR over time between groups. Please refer to Figure 1 legend for the abbreviations of the ANOVA table.

TEs compared to HMII without LLH $(P=0.025)$.

The incidence of hemolysis according to the INTERMACS definition was $13.6 \%$ in the LLH + sildenafil group, $50 \%$ in the LLH no sildenafil group, $8.8 \%$ in the no LLH +sildenafil group, and $13.3 \%$ in the no LLH no sildenafil group $(P=0.011)$.

The incidences of HS and GIB did not differ significantly between the 4 groups (Table IV).

The 1 year estimated survival free from TE (IS or PT) in the LLH + sildenafil group was $95 \pm 4 \%$ compared to $32 \pm 15 \%$ in the LLH no sildenafil and $92 \pm 4 \%$ in the no LLH + sildenafil group $(P=0.0001$, log-rank (MantelCox) test) (Figure 3A). All groups had a similar INR during the follow-up (Figure 2).

Cox regression analysis revealed a significantly higher risk for TE among HMII patients with LLH not taking sildenafil compared to LLH + sildenafil patients (HR: 14.4, 95\%-CI: 1.8 - 117.7, $P=0.001$ ) and compared to no LLH no sildenafil patients (HR: 11.5, 95\%-CI: 2.1 $31.7, P=0.043)$. The HR was reduced in LLH + sildenafil patients compared to LLH no sildenafil patients (HR: 1.8, 95\%-CI: $1.8-117.7, P=0.013)$. There was no difference in the survival free from gastrointestinal bleeding (GIB) in all 4 groups $(P=0.369)$ (Figure 3B). The overall survival regardless of AEs did not differ between the 4 groups $(P=0.110)$ (Figure $3 C)$.

Hemodynamic parameters: Patients who received sildenafil (LLH + sildenafil and no LLH + sildenafil groups) had significantly higher mPAP, PVR and PCWP compared to HMII patients who did not receive sildenafil (Tables I and V). On the other hand, cardiac index (CI), TAPSE and RV-FAC did not differ between all 4 groups preoperatively.

mPAP, PCWP decreased significantly 1-year postoperative in all groups but not in the no LLH no sildenafil group (Table V).

The mean difference of mPAP comparing pre- and postoperative measurements in the LLH + sildenafil group was $7.2 \pm 1.2 \mathrm{mmHg}(30.2 \pm 0.9$ versus $22.3 \pm 6.9$ $\mathrm{mmHg}, P<0.0001)$, and in the no LLH + sildenafil group, the mean difference in mPAP was $7.1 \pm 1.1 \mathrm{mmHg}$ $(34.3 \pm 9.7$ versus $28.5 \pm 7.2, P<0.0001)$ (Table V). In contrast, the mean difference in mPAP in the LLH no sildenafil group was only $4 \pm 1.4 \mathrm{mmHg}$ (Table V). PCWP also decreased significantly when comparing preoperative with 1 year post-implantation measurements, but 
Table III. Von Willebrand Factor Parameter Measurements

\begin{tabular}{lccccc}
\hline & $\begin{array}{c}\text { LLH + Sildenafil } \\
(n=22)\end{array}$ & $\begin{array}{c}\text { LLH no Sildenafil } \\
(n=12)\end{array}$ & $\begin{array}{c}\text { no LLH + Sildenafil } \\
(n=34)\end{array}$ & $\begin{array}{c}\text { no LLH no Sildenafil } \\
(n=15)\end{array}$ & $P$ value \\
\hline vWF: Ag \% & $236(116,654)$ & $148(126,278)$ & $204(94,284)$ & $233(114,720)$ & 0.558 \\
vWF: RCo & $143(75,180)$ & $102(85,147)$ & $125(71,173)$ & $115(85,302)$ & 0.594 \\
vWF: CBA & $193(85,302)$ & $115(103,166)$ & $130(58,240)$ & $166(126,278)$ & 0.698 \\
vWF: RCo/vWF: Ag & $0.58 \pm 0.12$ & $0.59 \pm 0.18$ & $0.64 \pm 0.14$ & $0.53 \pm 0.17$ & 0.269 \\
vWF: CBA/vWF: Ag & $0.72 \pm 0.15$ & $0.67 \pm 0.11$ & $0.71 \pm 0.19$ & $0.69 \pm 0.13$ & 0.716 \\
\hline vWF ind
\end{tabular}

vWF indicates von Willebrand factor; Ag, antigen; CBA, collagen binding activity; RCo, ristocetin cofactor activity; and HMWM, high-molecular-weight multimers.

Table IV. Distribution of Adverse Events Between Groups

\begin{tabular}{lccccc}
\hline & $\begin{array}{c}\text { LLH + Sildenafil } \\
(n=22)\end{array}$ & $\begin{array}{c}\text { LLH no Sildenafil } \\
(n=12)\end{array}$ & $\begin{array}{c}\text { no LLH + Sildenafil } \\
(n=34)\end{array}$ & $\begin{array}{c}\text { no LLH no Sildenafil } \\
(n=15)\end{array}$ & $P$-value \\
\hline PT $n(\%)$ & 0 & $3(25)$ & $1(2.9)$ & 0 & 0.005 \\
IS $n(\%)$ & 0 & $4(33.3)$ & $3(8.8)$ & 0 & 0.004 \\
TIA & $2(9.1)$ & $2(16.7)$ & 0 & $1(6.7)$ & 0.764 \\
HS & $1(4.5)$ & 0 & $2(5.9)$ & $1(6.7)$ & 0.843 \\
GIB & $6(27.3)$ & $4(33.3)$ & $16(17.6)$ & $5(33.3)$ & 0.473 \\
Hemolysis $n(\%)$ & $3(13.6)$ & $6(50)$ & $3(8.8)$ & $2(13.3)$ & 0.011 \\
\hline
\end{tabular}

PT indicates pump thrombosis; IS, ischemic stroke; HS, hemorrhagic stroke; GIB, gastrointestinal bleeding; and TIA, transitory ischemic attack.

again the mean decrease of PCWP values was higher in the sildenafil groups $(7.2 \mathrm{mmHg})$ compared to the no sildenafil groups $(4.3 \mathrm{mmHg})$ (Table $\mathrm{V})$.

PVR did not decrease significantly when comparing pre- and postoperative measurements in HMII patients, who did not have sildenafil (LLH no sildenafil and no LLH no sildenafil groups) (Table V). On the other hand, PVR decreased significantly 1-year after implantation in both sildenafil groups; $\mathrm{LLH}+$ sildenafil $(2.5 \pm 0.8$ versus $1.7 \pm 0.5$ Wood units, $P<0.0001)$ and no LLH + sildenafil $(3.0 \pm 1.1$ versus $2.1 \pm 0.9$ Wood units, $P<0.0001)$ (Table V). CI increased in all groups (Table V) but the mean increase was higher in HMII patients, who had sildenafil $\left(0.5 \mathrm{~L} /\right.$ minute $\left./ \mathrm{m}^{2}\right)$ (Table V). TAPSE increased significantly 1-year after HMII implantation only in the no LLH + sildenafil group. RV-FAC increased significantly 1 year post-implantation only in both sildenafil groups (Table V).

\section{Discussion}

Our study examined the clinical effect of LLH in patients on HMII support, as assessed by elevated LDH, and the association between sildenafil and TE. In our study, we found that patients on HMII support with LLH had a higher risk for TE and higher incidence of hemolysis and that the use of sildenafil in HMII with LLH reduced the risk of TE and sildenafil improved the pulmonary circulation significantly during HMII support regardless of LLH status. Similar results were described by Saeed, et $a l^{21)}$ who found that HMII patients with LLH not on sildenafil had a higher risk (HR 8.8) for TE compared to HMII with LLH on sildenafil. The difference in TE between the groups was not related to the variation in anticoagulation, as INR did not differ between all 4 groups. PDE5-Is intensify the effect of endogenous NO by stabilizing the second-messengers cyclic adenylate monophosphate (cAMP) and cyclic guanylate monophosphate (cGMP). ${ }^{22)}$ cGMP activates the cGMP-dependent protein kinases (cGKs) and also inhibits phosphodiesterase 3, and thus inhibits the destruction of cyclic adenosine monophosphate (cAMP). cAMP also activates the cAMP-dependent protein kinases (PKA), which are known platelet inhibitors. The NO/cGMP-induced platelet inhibition can also be resolved by both cGK- and PKA-dependent mechanisms. ${ }^{23-25)}$ However, it is still unclear how cGKs mediate the platelet-inhibitory effect of the NO/cGMP. Many hypotheses have been presented and it has been shown that NO/cGMP inhibits intracellular calcium release, ${ }^{26)}$ which is an important step to full activation of platelets. ${ }^{27)}$ It was unclear how NO/cGMP resolve this effect but recently Antl, et $a l^{28)}$ demonstrated that cGK phosphorylates inositol-1,4,5-trisphosphate receptor-associated cGMP kinase substrate, which leads to inhibition of intracellular calcium release.

Our hypothesis was that sildenafil, a PDE5-I, which is usually prescribed to CF-LVAD patients to treat pulmonary hypertension and right heart failure, might reduce the risk of TE in HMII patients with LLH, due to enhanced NO/cGMP signaling. In addition to a reduction of TE incidence in HMII patients on sildenafil, we detected that $\mathrm{LDH}$ and $\mathrm{fHb}$ decreased in LLH patients over time in comparison to LLH patients not on sildenafil. This effect might be a result of the hemodynamic effect of sildenafil since it lowers pulmonary vascular resistance and pulmonary capillary wedge pressure and increases LV preload without causing systemic hypotension ${ }^{29-31}$ and therefore leads to an increase in LVAD flow, which in turn may have contributed to lower TE due to better flow through the device.

In our study, we found decreases in mPAP and PCWP and an increase of CI in all HMII patients 1 year 

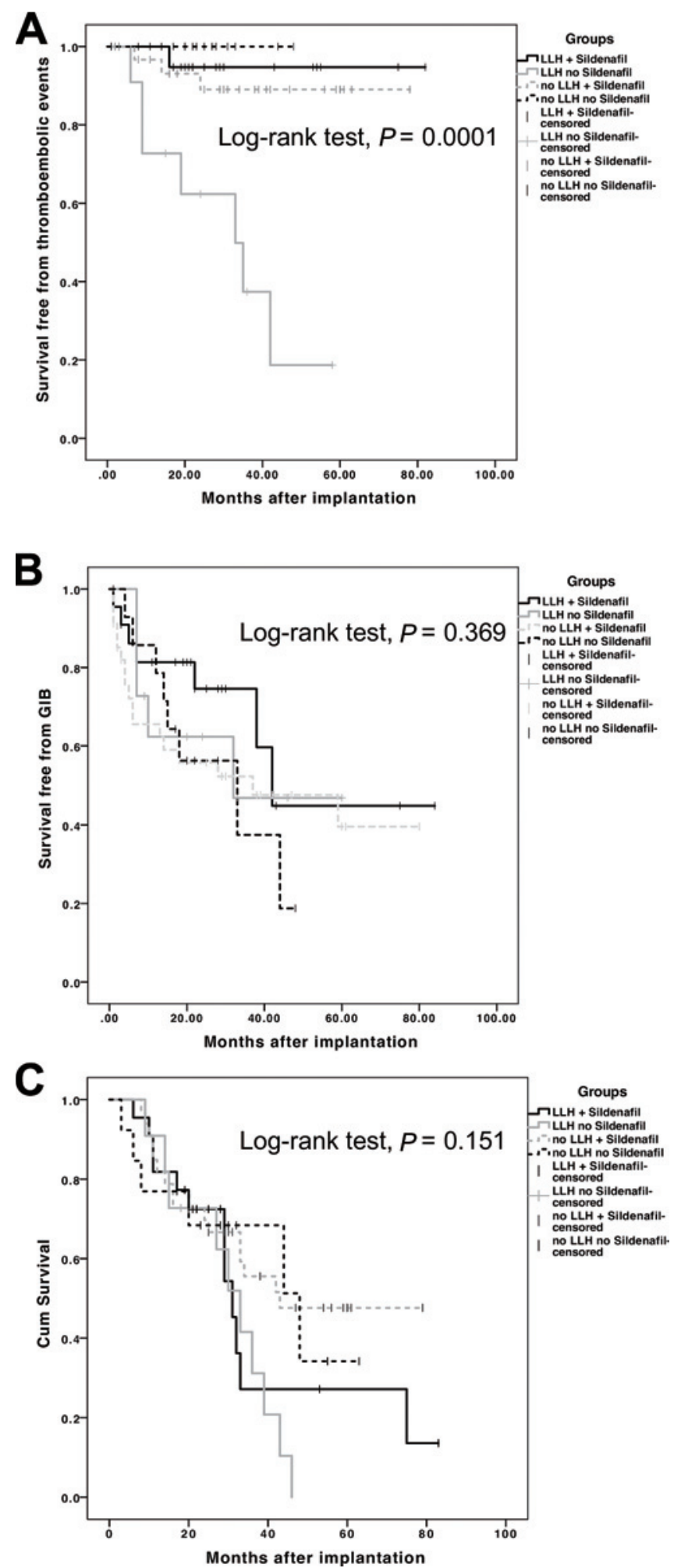

Figure 3. Kaplan-Meier curves: Overall survival and survival free from thromboembolic events and GIB. A: Survival free from thromboembolic events. B: Survival free from GIB. C: Overall survival of all 4 groups. GIB indicates gastrointestinal bleeding. 
Table V. Pre- and One-Year Postoperative Hemodynamic Parameters

\begin{tabular}{|c|c|c|c|c|c|c|c|c|}
\hline & $\begin{array}{l}\text { 1. LLH + } \\
\text { Sildenafil } \\
(n=22)\end{array}$ & $\begin{array}{l}\text { 2. LLH no } \\
\text { Sildenafil } \\
(n=12)\end{array}$ & $\begin{array}{c}P \text {-value } \\
1 \text { versus } 2\end{array}$ & $\begin{array}{c}P \text {-value } \\
1 \text { versus } 3\end{array}$ & $\begin{array}{l}\text { 3. no LLH } \\
\text { + Sildenafil } \\
(n=34)\end{array}$ & $\begin{array}{c}P \text {-value } \\
2 \text { versus } 3\end{array}$ & $\begin{array}{l}\text { 4. no LLH no } \\
\text { Sildenafil } \\
(n=15)\end{array}$ & $\begin{array}{c}P \text {-value } \\
3 \text { versus } 4\end{array}$ \\
\hline \multicolumn{9}{|l|}{ mPAP mmHg } \\
\hline Pre & $30.2 \pm 8.9$ & $22.6 \pm 3.8$ & $0.020 *$ & 0.145 & $34.3 \pm 9.7$ & $<0.0001 *$ & $21.4 \pm 7.7$ & $<0.001 *$ \\
\hline post & $22.3 \pm 6.9$ & $18.9 \pm 3.3$ & 0.315 & 0.138 & $28.5 \pm 7.2$ & $<0.0001 *$ & $18.9 \pm 5.7$ & $0.008 *$ \\
\hline$P$-value pre versus post & $<0.0001 *$ & $0.029 *$ & & & $<0.0001 *$ & & 0.222 & \\
\hline \multicolumn{9}{|l|}{ PCWP mmHg } \\
\hline pre & $22.2 \pm 6.3$ & $16.1 \pm 1.5$ & $0.027 *$ & 0.998 & $21.1 \pm 9.3$ & $0.032 *$ & $14.3 \pm 5.6$ & $<0.001^{*}$ \\
\hline post & $14.9 \pm 5.8$ & $11.3 \pm 2.8$ & 0.319 & 0.465 & $17.5 \pm 5.8$ & $0.018^{*}$ & $9.1 \pm 2.8$ & $0.009 *$ \\
\hline$P$-value pre versus post & $<0.0001$ & $<0.01 *$ & & & $<0.001 *$ & & $<0.01 *$ & \\
\hline \multicolumn{9}{|l|}{ PVR Wood units } \\
\hline pre & $2.5 \pm 0.8$ & $1.4 \pm 0.4$ & $0.005^{*}$ & 0.224 & $3.0 \pm 1.1$ & $<0.001^{*}$ & $1.5 \pm 0.9$ & $<0.001 *$ \\
\hline post & $1.7 \pm 0.5$ & $1.2 \pm 0.5$ & 0.308 & 0.646 & $2.1 \pm 0.9$ & $0.034 *$ & $1.2 \pm 0.6$ & $0.031 *$ \\
\hline$P$-value pre versus post & $<0.0001 *$ & 0.776 & & & $<0.0001^{*}$ & & 0.680 & \\
\hline \multicolumn{9}{|l|}{$\mathrm{CI} \mathrm{L} / \mathrm{minute} / \mathrm{m}^{2}$} \\
\hline pre & $2.0 \pm 0.4$ & $2.1 \pm 0.3$ & 0.999 & 0.974 & $1.9 \pm 0.4$ & 0.988 & $1.9 \pm 0.3$ & 0.999 \\
\hline post & $2.5 \pm 0.5$ & $2.3 \pm 0.2$ & 0.326 & 0.146 & $2.4 \pm 0.5$ & 0.994 & $2.3 \pm 0.2$ & 0.369 \\
\hline$P$-value pre versus post & $<0.0001^{*}$ & $0.040 *$ & & & $<0.0001 *$ & & $0.010 *$ & \\
\hline \multicolumn{9}{|l|}{ TAPSE $\mathrm{mm}$} \\
\hline pre & $15.3 \pm 4.4$ & $16.8 \pm 2.1$ & 0.566 & 0.634 & $14.3 \pm 2.3$ & 0.089 & $16.2 \pm 2.8$ & 0.204 \\
\hline post & $16.4 \pm 2.5$ & $17.1 \pm 1.4$ & 0.931 & 0.285 & $15.3 \pm 2.4$ & 0.150 & $17.5 \pm 2.3$ & $0.034 *$ \\
\hline$P$-value pre versus post & 0.219 & 0.994 & & & $0.011 *$ & & 0.357 & \\
\hline \multicolumn{9}{|l|}{ RV-FAC \% } \\
\hline pre & $36.9 \pm 10.2$ & $39.4 \pm 9.4$ & 0.897 & 0.941 & $35.4 \pm 9.2$ & 0.615 & $40.3 \pm 9.6$ & 0.361 \\
\hline post & $41.5 \pm 7.6$ & $38.9 \pm 8.2$ & 0.764 & 0.610 & $38.6 \pm 6.2$ & 0.999 & $41.7 \pm 7.7$ & 0.667 \\
\hline$P$-value pre versus post & $0.002 *$ & 0.983 & & & $0.009 *$ & & 0.856 & \\
\hline
\end{tabular}

mPAP indicates mean pulmonary artery pressure; PCWP, pulmonary capillary wedge pressure; PVR, pulmonary vascular resistance; CI, cardiac index; TAPSE, tricuspid annular plane systolic excursion; and RV-FAC, right ventricle fractional area change. Data are presented as the mean \pm SD. Comparisons of repeated measurements between groups were conducted with 2-way ANOVA with Tukey's correction. *indicates significance.

after implantation, as a result of the decompression of pulmonary circulation during HMII support, results that were similar to those noted by Alturi, et $a l .^{32)}$ The mean increase of CI and decreases of MPAP and PCWP were higher in HMII, who had sildenafil.

In addition, PVR decreased significantly 1-year postimplantation only in HMII patients, who had sildenafil and also the RV-FAC increased significantly in these patients. According to our findings and in accordance with previous studies, ${ }^{18-20)}$ we can conclude that the use of sildenafil during HMII supports add a further decompression effect of the pulmonary circulation during HMII support providing a better hemodynamic effect. These findings strengthen the above-mentioned hypothesis about the hemodynamic effect of sildenafil which might contribute to the lower TE incidence in HMII patients with LLH receiving sildenafil.

The etiology of hemolysis in patients with CF-LVAD is still not fully understood; red blood cell damage might be the direct result of shear stress induced by the device itself. However, many other factors, such as oxidative stress, medications, and infections contribute to the process of hemolysis. ${ }^{4,33,34)} \mathrm{NO}$ is continuously synthesized in the endothelium and has many functions, such as the regulation of homeostatic vascular functions through vasodilation and inhibition of platelet activation and thrombosis. ${ }^{35,36)}$ Elevated $\mathrm{fHb}$, as it appears in hemolysis conditions, increases the scavenging of $\mathrm{NO}$ and enhances its consumption, resulting in several cellular dysfunctions, such as vasoconstriction, platelet activation and systemic and pulmonary hypertension, in patients with hemolytic disorders. ${ }^{13,35,37)}$ In our study, fHb remained significantly higher in LLH patients compared to no LLH patients, but $\mathrm{fHb}$ decreased significantly in LLH patients taking sildenafil compared to LLH patients not on sildenafil.

Elevated $\mathrm{fHb}$, which occurs in pump induced hemolysis, can also contribute to the pro-coagulation pathway via its interaction with vWF. fHb interacts with the A1 and 2 domains of VWF, which in turn increase the adhesion of platelets to VWF. ${ }^{15)}$ In our study, we could not detect a significant difference in the measured vWF parameters in HMII patients with LLH or between patients taking sildenafil and not taking sildenafil. The incidence of GIB also did not differ between the groups.

Limitations of the study: Our study is limited by the usual shortcomings of a small-cohort single-center study. Our data suffer potential bias in patient selection and data acquisition; therefore, caution should be taken when interpreting the results. Due to the low number of AEs, adjustment in the regression model was not performed to covariates, which may have an impact on TE, and accordingly, the estimated hazard ratio is under powered.

Hemolysis in LVAD patients is multifactorial, and as a result, we cannot exclude the possibility that unmeasured confounders influenced our results. Another limitation was the small number and heterogeneity of the patients, which may limit the generalization of the results. 


\section{Conclusion}

LLH in HMII patients as defined by elevated LDH is associated with higher thromboembolic rates. Sildenafil treatment among HMII patients with LLH reduced the risk of thromboembolic events. Sildenafil significantly improved the pulmonary circulation and added a further decompression effect during HMII support. Further investigation is needed to understand the pathophysiological role of PDE5-Is in the reduction of thromboembolic events among patients with CF-LVAD devices.

\section{Disclosures}

Conflicts of interest: The authors state that there are no conflicts of interest to declare.

\section{References}

1. Kirklin JK, Naftel DC, Pagani FD, et al. Seventh INTERMACS annual report: 15,000 patients and counting. J Heart Lung Transplant 2015; 34: 1495-504.

2. Slaughter MS, Rogers JG, Milano CA, et al. Advanced heart failure treated with continuous-flow left ventricular assist device. N Engl J Med 2009; 361: 2241-51.

3. Stulak JM, Davis ME, Haglund N, et al. Adverse events in contemporary continuous-flow left ventricular assist devices: A multi-institutional comparison shows significant differences. J Thorac Cardiovasc Surg 2016; 151: 177-89.

4. Katz JN, Jensen BC, Chang PP, Myers SL, Pagani FD, Kirklin JK. A multicenter analysis of clinical hemolysis in patients supported with durable, long-term left ventricular assist device therapy. J Heart Lung Transplant 2015; 34: 701-9.

5. Genovese EA, Dew MA, Teuteberg JJ, et al. Incidence and patterns of adverse event onset during the first 60 days after ventricular assist device implantation. Ann Thorac Surg 2009; 88: 1162-70.

6. Uriel N, Han J, Morrison KA, et al. Device thrombosis in HeartMate II continuous-flow left ventricular assist devices: a multifactorial phenomenon. J Heart Lung Transplant 2014; 33: 51-9.

7. Cowger JA, Romano MA, Shah P, et al. Hemolysis: a harbinger of adverse outcome after left ventricular assist device implant. J Heart Lung Transplant 2014; 33: 35-43.

8. INTERMACS. Protocol 3.0, Appendix A- Adverse Event Definitions version 2.22013 updated 12.04.2013. Available at: https:// www.uab.edu/medicine/intermacs/appendices/app-a-5-0

9. Levin AP, Saeed O, Willey JZ, et al. Watchful waiting in continuous-flow left ventricular assist device patients with ongoing hemolysis is associated with an increased risk for cerebrovascular accident or death. Circ Heart Fail 2016; 9: e002896.

10. Shah P, Mehta VM, Cowger JA, Aaronson KD, Pagani FD. Diagnosis of hemolysis and device thrombosis with lactate dehydrogenase during left ventricular assist device support. J Heart Lung Transplant 2014; 33: 102-4.

11. Tran PL, Pietropaolo MG, Valerio L, et al. Hemolysate-mediated platelet aggregation: an additional risk mechanism contributing to thrombosis of continuous flow ventricular assist devices. Perfusion 2016; 31: 401-8.

12. Sugiura T, Okumiya T, Kubo T, Takeuchi H, Matsumura $Y$. Evaluation of intravascular hemolysis with erythrocyte creatine in patients with aortic stenosis. Int Heart J 2016; 57: 430-3.

13. Donadee C, Raat NJ, Kanias T, et al. Nitric oxide scavenging by red cell microparticles and cell free hemoglobin as a mechanism for the red cell storage lesion. Circulation 2011; 124: 465-76.

14. Sertorio JT, Neto-Neves EM, Dias-Junior CA, et al. Elevated plasma hemoglobin levels increase nitric oxide consumption in experimental and clinical acute pulmonary thromboembolism. Crit Care Med 2013; 41: e118-24.

15. Da Q, Teruya M, Guchhait P, Teruya J, Olson JS, Cruz MA Free hemoglobin increases von Willebrand factor-mediated platelet adhesion in vitro: implications for circulatory devices. Blood 2015; 126: 2338-41.

16. Schermuly RT, Krupnik E, Tenor H, et al. Coaerosolization of phosphodiesterase inhibitors markedly enhances the pulmonary vasodilatory response to inhaled iloprost in experimental pulmonary hypertension. Maintenance of lung selectivity. Am J Respir Crit Care Med 2001; 164: 1694-700.

17. Weimann J, Ullrich R, Hromi J, et al. Sildenafil is a pulmonary vasodilator in awake lambs with acute pulmonary hypertension. Anesthesiology 2000; 92: 1702-12.

18. Baker WL, Radojevic J, Gluck JA. Systematic review of phosphodiesterase-5 inhibitor use in right ventricular failure following left ventricular assist device implantation. Artif Organs 2016; 40: 123-8.

19. Hamdan R, Mansour H, Nassar P, Saab M. Prevention of right heart failure after left ventricular assist device implantation by phosphodiesterase 5 inhibitor. Artif Organs 2014; 38: 963-7.

20. Tedford RJ, Hemnes AR, Russell SD, et al. PDE5A inhibitor treatment of persistent pulmonary hypertension after mechanical circulatory support. Circ Heart Fail 2008; 1: 213-9.

21. Saeed O, Rangasamy S, Selevany I, et al. Sildenafil is associated with reduced device thrombosis and ischemic stroke despite low-level hemolysis on Heart Mate II support. Circ Heart Fail 2017; 10: e004222.

22. Ahn HS, Foster M, Cable M, Pitts BJ, Sybertz EJ. Ca/CaMstimulated and cGMP-specific phosphodiesterases in vascular and non-vascular tissues. Adv Exp Med Biol 1991; 308: 191-7.

23. Marjanovic JA, Li Z, Stojanovic A, Du X. Stimulatory roles of nitric-oxide synthase 3 and guanylyl cyclase in platelet activation. J Biol Chem 2005; 280: 37430-8.

24. $\mathrm{Li} \mathrm{Z}, \mathrm{Xi} \mathrm{X}, \mathrm{Gu} \mathrm{M}$, et al. A stimulatory role for cGMPdependent protein kinase in platelet activation. Cell 2003; 112: 77-86.

25. Jang EK, Azzam JE, Dickinson NT, Davidson MM, Haslam RJ. Roles for both cyclic GMP and cyclic AMP in the inhibition of collagen-induced platelet aggregation by nitroprusside. $\mathrm{Br} \mathrm{J}$ Haematol 2002; 117: 664-75.

26. Hofmann F. The biology of cyclic GMP-dependent protein kinases. J Biol Chem 2005; 280: 1-4.

27. Jackson SP, Nesbitt WS, Kulkarni S. Signaling events underlying thrombus formation. J Thromb Haemost 2003; 1: 1602-12.

28. Antl M, von Bruhl ML, Eiglsperger C, et al. IRAG mediates NO/cGMP-dependent inhibition of platelet aggregation and thrombus formation. Blood 2007; 109: 552-9.

29. Lewis GD, Lachmann J, Camuso J, et al. Sildenafil improves exercise hemodynamics and oxygen uptake in patients with systolic heart failure. Circulation 2007; 115: 59-66.

30. Lepore JJ, Maroo A, Bigatello LM, et al. Hemodynamic effects of sildenafil in patients with congestive heart failure and pulmonary hypertension: combined administration with inhaled nitric oxide. Chest 2005; 127: 1647-53.

31. Igarashi A, Inoue S, Ishii T, Tsutani K, Watanabe H. Comparative effectiveness of oral medications for pulmonary arterial hypertension. Int Heart J 2016; 57: 466-72.

32. Atluri P, Fairman AS, MacArthur JW, et al. Continuous flow left ventricular assist device implant significantly improves pulmonary hypertension, right ventricular contractility, and tricuspid valve competence. J Card Surg 2013; 28: 770-5.

33. Yasuda T, Shimokasa K, Funakubo A, Higami T, Kawamura T, Fukui Y. An investigation of blood flow behavior and hemolysis in artificial organs. ASAIO J 2000; 46: 527-31.

34. Alhosaini H, Jensen BC, Chang PP, Sheridan BC, Katz JN. A novel link between G6PD deficiency and hemolysis in patients with continuous-flow left ventricular assist devices. J Heart Lung Transpl 2014; 33: 116-7. 
35. Minneci PC, Deans KJ, Zhi H, et al. Hemolysis-associated endothelial dysfunction mediated by accelerated NO inactivation by decompartmentalized oxyhemoglobin. J Clin Invest 2005; 115: 3409-17.

36. Palmer RM, Ferrige AG, Moncada S. Nitric oxide release ac- counts for the biological activity of endothelium-derived relaxing factor. Nature 1987; 327: 524-6.

37. Zhou Z, Behymer M, Guchhait P. Role of extracellular hemoglobin in thrombosis and vascular occlusion in patients with sickle cell anemia. Anemia 2011; 2011: 918916. 